\title{
Supporting Details:
}

Crystal Structures and Magnetic Properties of Mixed Iridium-Ruthenium Triple Perovskites: $\mathrm{Ba}_{3} \mathrm{MRulrO}_{9}$ Part I: $(\mathrm{M}=$ Lanthanide, $\mathrm{Y})$

\section{Synthetic Details}

Starting reactants were $\mathrm{RuO}_{2}$, Ir (Engelhard, 99.5\%), $\mathrm{BaCO}_{3}$ (Alfa Aesar, 99.95\%), $\mathrm{Y}_{2} \mathrm{O}_{3}$ (Alfa Aesar, $99.99 \%$ ), $\mathrm{La}_{2} \mathrm{O}_{3}$ (Alfa Aesar, 99.9\%), $\mathrm{CeO}_{2}$ (Alfa Aesar, 99.99\%), $\operatorname{Pr}_{6} \mathrm{O}_{11}$ (Alfa Aesar, 99.9\%), $\mathrm{Nd}_{2} \mathrm{O}_{3}$ (Alfa Aesar, $99.9 \%$ ), $\mathrm{Sm}_{2} \mathrm{O}_{3}$ (Alfa Aesar, 99.9\%), $\mathrm{Eu}_{2} \mathrm{O}_{3}$ (Alfa Aesar, 99.9\%), $\mathrm{Gd}_{2} \mathrm{O}_{3}$ (Alfa Aesar, 99.9\%), $\mathrm{Tb}_{4} \mathrm{O}_{7}$ (Alfa Aesar, 99.9\%), $\mathrm{Dy}_{2} \mathrm{O}_{3}$ (Alfa Aesar, 99.9\%), $\mathrm{Ho}_{2} \mathrm{O}_{3}$ (Alfa Aesar, 99.9\%), $\mathrm{Er}_{2} \mathrm{O}_{3}$ (Alfa Aesar, 99.9\%), $\mathrm{Tm}_{2} \mathrm{O}_{3}$ (Alfa Aesar, 99.9\%), $\mathrm{Yb}_{2} \mathrm{O}_{3}$ (Alfa Aesar, 99.9\%), and $\mathrm{Lu}_{2} \mathrm{O}_{3}$ (Alfa Aesar, 99.9\%).

Successive heating, to the temperature given in parenthesis, and grinding cycles were performed in air for $\mathrm{Ba}_{3} \mathrm{YRuIrO}_{9}\left(1225^{\circ} \mathrm{C}\right), \mathrm{Ba}_{3} \mathrm{LaRuIrO}_{9}\left(1100{ }^{\circ} \mathrm{C}\right), \mathrm{Ba}_{3} \mathrm{PrRuIrO}_{9}(1125$ $\left.{ }^{\circ} \mathrm{C}\right), \mathrm{Ba}_{3} \mathrm{NdRuIrO}_{9}\left(1125^{\circ} \mathrm{C}\right), \mathrm{Ba}_{3} \mathrm{SmRuIrO}_{9}\left(1125^{\circ} \mathrm{C}\right), \mathrm{Ba}_{3} \mathrm{EuRuIrO}_{9}\left(1125^{\circ} \mathrm{C}\right)$, $\mathrm{Ba}_{3} \mathrm{GdRuIrO}_{9}\left(1125^{\circ} \mathrm{C}\right), \mathrm{Ba}_{3} \mathrm{DyRuIrO}_{9}\left(1150^{\circ} \mathrm{C}\right), \mathrm{Ba}_{3} \mathrm{MgRuIrO}_{9}\left(1100^{\circ} \mathrm{C}\right)$, $\mathrm{Ba}_{3} \mathrm{HoRuIrO}_{9}\left(1200^{\circ} \mathrm{C}\right), \mathrm{Ba}_{3} \mathrm{ErRuIrO}_{9}\left(1225^{\circ} \mathrm{C}\right), \mathrm{Ba}_{3} \mathrm{TmRuIrO}_{9}\left(1225^{\circ} \mathrm{C}\right)$, $\mathrm{Ba}_{3} \mathrm{YbRuIrO}_{9}\left(1200{ }^{\circ} \mathrm{C}\right)$ and $\mathrm{Ba}_{3} \mathrm{LuRuIrO}_{9}\left(1200{ }^{\circ} \mathrm{C}\right)$. 
Table A - Atomic coordinates are $\operatorname{Ba} 1(0,0,1 / 4), \operatorname{Ba} 2(1 / 3,2 / 3, z), \operatorname{M}(0,0,0), \operatorname{Ru} / \operatorname{Ir}(1 / 3,2 / 3, z)$, $\mathrm{O} 1(x, 2 x, 1 / 4), \mathrm{O} 2(x, 2 x, z)$ in space group $\mathrm{P}_{3} / m m c$. Isotropic thermal parameters and refinement details are given. Background subtracted $\mathrm{R}_{\mathrm{wp}}$ and $\mathrm{R}_{\mathrm{p}}$ are given.

\begin{tabular}{|c|c|c|c|c|c|}
\hline & $\mathrm{Ba}_{3} \mathrm{YRuIrO}_{9}$ & $\mathrm{Ba}_{3} \mathrm{InRuIrO}{ }_{9}$ & $\mathrm{Ba}_{3} \mathrm{LaRuIrO}_{9}$ & $\mathrm{Ba}_{3} \mathrm{PrRuIrO}_{9}$ & $\mathrm{Ba}_{3} \mathrm{NdRuIrO}_{9}$ \\
\hline $\mathrm{a}(\AA)$ & $5.8846(3)$ & $5.8213(4)$ & $5.9588(8)$ & $5.8892(2)$ & $5.93750(29)$ \\
\hline $\mathrm{c}(\AA)$ & $14.5494(9)$ & $14.3648(12)$ & $15.0928(22)$ & $14.6345(6)$ & $14.8177(8)$ \\
\hline Vol. $\left(\AA^{3}\right)$ & $436.33(4)$ & $421.57(5)$ & $464.11(12)$ & $439.56(3)$ & $452.40(4)$ \\
\hline $\mathrm{Ba} 2 z$ & $0.90487(17)$ & $0.9105(2)$ & $0.8952(2)$ & $0.90230(14)$ & $0.89995(16)$ \\
\hline $\mathrm{Ru} / \mathrm{Ir} z$ & $0.16217(14)$ & $0.16006(19)$ & $0.16504(18)$ & $0.16466(12)$ & $0.16441(14)$ \\
\hline O1x & $0.5084(15)$ & $0.526(2)$ & $0.510(2)$ & $0.5154(16)$ & $0.5136(17)$ \\
\hline $\mathrm{O} 2 x$ & $0.1648(15)$ & $0.1564(19)$ & $0.1710(17)$ & $0.1721(14)$ & $0.1710(15)$ \\
\hline $\mathrm{O} 2 z$ & $0.4142(8)$ & $0.4127(11)$ & $0.4086(8)$ & $0.4152(7)$ & $0.4108(7)$ \\
\hline $\mathrm{Ui} / \mathrm{Ue}^{*} 100 \mathrm{Ba} 1$ & $0.08(14)$ & $-0.13(18)$ & $0.0(2)$ & $0.36(11)$ & $0.05(12)$ \\
\hline $\mathrm{Ui} / \mathrm{Ue}^{*} 100 \mathrm{Ba} 2$ & $0.73(9)$ & $0.53(10)$ & $0.75(11)$ & $1.17(7)$ & $0.99(8)$ \\
\hline $\mathrm{Ui} / \mathrm{Ue}^{*} 100 \mathrm{M}$ & $-0.7(2)$ & $0.47(24)$ & $0.54(19)$ & $0.14(11)$ & $-0.1(1)$ \\
\hline $\mathrm{Ui} / \mathrm{Ue}^{*} 100 \mathrm{Ru} / \mathrm{Ir}$ & $0.25(7)$ & $-0.25(8)$ & $0.1(1)$ & $0.44(6)$ & $0.12(6)$ \\
\hline Ui/Ue*100 O1 & $-1.8(5)$ & $3.3(11)$ & $1.3(10)$ & $2.8(7)$ & $1.4(7)$ \\
\hline $\mathrm{Ui} / \mathrm{Ue}^{*} 100 \mathrm{O} 2$ & $-0.7(4)$ & $-1.8(5)$ & $-1.9(4)$ & $0.1(3)$ & $-0.6(3)$ \\
\hline $\mathrm{R}_{\mathrm{wp}}(\%)$ & 16.57 & 16.00 & 14.86 & 14.27 & 15.08 \\
\hline $\mathrm{R}_{\mathrm{W}}(\%)$ & 12.11 & 12.45 & 11.72 & 10.83 & 12.02 \\
\hline $\mathrm{R}\left(\mathrm{F}^{2}\right)$ & 8.52 & 9.01 & 8.06 & 6.93 & 8.83 \\
\hline$\chi^{2}$ & 3.31 & 3.46 & 2.91 & 2.27 & 2.61 \\
\hline & $\mathrm{Ba}_{3} \mathrm{SmRuIrO}_{9}$ & $\mathrm{Ba}_{3} \mathrm{EuRuIrO}_{9}$ & $\mathrm{Ba}_{3} \mathrm{GdRuIrO}_{9}$ & $\mathrm{Ba}_{3} \mathrm{TbRuIrO}_{9}$ & $\mathrm{Ba}_{3} \mathrm{DyRuIrO}_{9}$ \\
\hline $\mathrm{a}(\AA)$ & $5.9231(3)$ & $5.9169(3)$ & $5.9115(2)$ & $5.8452(3)$ & $5.8938(3)$ \\
\hline$c(\AA)$ & $14.7253(7)$ & $14.6941(8)$ & $14.6600(6)$ & $14.4697(7)$ & $14.5841(7)$ \\
\hline Vol. $\left(\AA^{3}\right)$ & $447.393(35)$ & $445.51(4)$ & $443.669(31)$ & $428.136(33)$ & $438.74(3)$ \\
\hline $\mathrm{Ba} 2 z$ & $0.90229(15)$ & $0.90292(15)$ & $0.90327(16)$ & $0.90594(17)$ & $0.90452(15)$ \\
\hline $\mathrm{Ru} / \mathrm{Ir} z$ & $0.16382(13)$ & $0.16359(13)$ & $0.16345(15)$ & $0.16249(14)$ & $0.16261(13)$ \\
\hline O1x & $0.5146(17)$ & $0.5164(17)$ & $0.5135(17)$ & $0.5087(18)$ & $0.5154(16)$ \\
\hline $\mathrm{O} 2 x$ & $0.1733(14)$ & $0.1691(14)$ & $0.1699(16)$ & $0.1657(17)$ & $0.1667(15)$ \\
\hline $\mathrm{O} 2 z$ & $0.4118(7)$ & $0.4125(7)$ & $0.4126(8)$ & $0.4135(8)$ & $0.4126(7)$ \\
\hline Ui/Ue*100 Ba1 & $0.0(1)$ & $0.3(1)$ & $-0.2(1)$ & $-0.1(1)$ & $-0.1(1)$ \\
\hline $\mathrm{Ui} / \mathrm{Ue}^{*} 100 \mathrm{Ba} 2$ & $0.98(8)$ & $1.18(8)$ & $1.13(9)$ & $0.68(9)$ & $1.03(8)$ \\
\hline $\mathrm{Ui} / \mathrm{Ue}^{*} 100 \mathrm{M}$ & $0.0(1)$ & $0.0(1)$ & $-0.46(13)$ & $0.0(1)$ & $-0.1(1)$ \\
\hline $\mathrm{Ui} / \mathrm{Ue}^{*} 100 \mathrm{Ru} / \mathrm{Ir}$ & $0.29(6)$ & $0.41(6)$ & $0.58(7)$ & $0.12(7)$ & $0.35(6)$ \\
\hline $\mathrm{Ui} / \mathrm{Ue}^{*} 100 \mathrm{O} 1$ & $2.1(8)$ & $2.0(7)$ & $0.4(7)$ & $0.7(7)$ & $1.1(7)$ \\
\hline $\mathrm{Ui} / \mathrm{Ue}^{*} 100 \mathrm{O} 2$ & $-1.1(3)$ & $-0.6(3)$ & $-0.8(3)$ & $-1.2(4)$ & $-0.7(3)$ \\
\hline $\mathrm{R}_{\mathrm{wp}}(\%)$ & 14.55 & 14.28 & 16.97 & 16.76 & 14.67 \\
\hline $\mathrm{R}_{\mathrm{wp}}(\%)$ & 11.58 & 11.48 & 13.40 & 13.55 & 11.71 \\
\hline $\mathrm{R}\left(\mathrm{F}^{2}\right)$ & 7.85 & 7.71 & 9.90 & 9.50 & 8.27 \\
\hline$\chi^{2}$ & 2.47 & 2.61 & 3.29 & 3.05 & 2.70 \\
\hline
\end{tabular}




\begin{tabular}{llllll}
\hline & $\mathrm{Ba}_{3} \mathrm{HoRuIrO}_{9}$ & $\mathrm{Ba}_{3} \mathrm{ErRuIrO}_{9}$ & $\mathrm{Ba}_{3} \mathrm{TmRuIrO}_{9}$ & $\mathrm{Ba}_{3} \mathrm{YbRuIrO}_{9}$ & $\mathrm{Ba}_{3} \mathrm{LuRuIrO}_{9}$ \\
\hline $\mathrm{a}(\AA)$ & $5.8866(2)$ & $5.8776(3)$ & $5.8728(2)$ & $5.86362(22)$ & $5.8580(3)$ \\
$\mathrm{c}(\AA)$ & $14.5576(6)$ & $14.5277(9)$ & $14.5041(6)$ & $14.4793(6)$ & $14.4644(7)$ \\
$\mathrm{Vol} .\left(\AA^{3}\right)$ & $436.87(3)$ & $434.63(4)$ & $433.22(3)$ & $431.13(3)$ & $429.87(3)$ \\
$\mathrm{Ba} 2$ & $0.90484(14)$ & $0.90517(15)$ & $0.90556(12)$ & $0.90638(14)$ & $0.90668(14)$ \\
$\mathrm{Ru} / \mathrm{Ir} z$ & $0.16228(12)$ & $0.16253(13)$ & $0.16241(10)$ & $0.16183(12)$ & $0.16165(12)$ \\
$\mathrm{O} 1 x$ & $0.5129(15)$ & $0.5091(15)$ & $0.4903(12)$ & $0.5164(15)$ & $0.5101(14)$ \\
$\mathrm{O} 2 x$ & $0.1668(13)$ & $0.1688(15)$ & $0.1750(12)$ & $0.1682(13)$ & $0.1677(13)$ \\
$\mathrm{O} 2 z$ & $0.4132(7)$ & $0.4121(7)$ & $0.4136(7)$ & $0.4133(7)$ & $0.4122(7)$ \\
$\mathrm{Ui} / \mathrm{Ue}^{*} 100 \mathrm{Ba} 1$ & $0.0(1)$ & $-0.3(1)$ & $1.11(11)$ & $0.12(12)$ & $0.28(12)$ \\
$\mathrm{Ui} / \mathrm{Ue} 100 \mathrm{Ba} 2$ & $0.86(7)$ & $0.47(8)$ & $1.96(7)$ & $0.85(7)$ & $0.92(7)$ \\
$\mathrm{Ui} / \mathrm{Ue} 100 \mathrm{M}$ & $0.0(1)$ & $-0.40(12)$ & $1.15(11)$ & $0.20(11)$ & $0.41(10)$ \\
$\mathrm{Ui} / \mathrm{Ue} 100 \mathrm{Ru} / \mathrm{Ir}$ & $0.44(6)$ & $0.09(6)$ & $1.53(5)$ & $0.42(6)$ & $0.36(5)$ \\
$\mathrm{Ui} / \mathrm{Ue}$ 100 O1 & $0.9(6)$ & $-0.5(6)$ & $2.1(5)$ & $1.56(63)$ & $0.5(6)$ \\
$\mathrm{Ui} / \mathrm{Ue}$ 100 O2 & $-0.6(3)$ & $-1.1(3)$ & $2.5(3)$ & $-1.1(3)$ & $0.0(3)$ \\
$\mathrm{R}_{\mathrm{wp}}(\%)$ & 13.01 & 14.32 & 8.65 & 13.07 & 12.30 \\
$\mathrm{R}_{\mathrm{p}}(\%)$ & 10.32 & 11.40 & 6.81 & 10.48 & 9.39 \\
$\mathrm{R}\left(\mathrm{F}^{2}\right)$ & 7.96 & 8.55 & 5.99 & 8.09 & 6.34 \\
$\chi^{2}$ & 2.63 & 2.80 & 1.86 & 2.54 & 2.38 \\
\hline
\end{tabular}


Table B - Selected interatomic distances $(\AA)$.

\begin{tabular}{lllll}
\hline & $\mathrm{Ba}_{3} \mathrm{YRuIrO}_{9}$ & $\mathrm{Ba}_{3} \mathrm{LaRuIrO}_{9}$ & $\mathrm{Ba}_{3} \mathrm{PrRuIrO}_{9}$ & $\mathrm{Ba}_{3} \mathrm{NdRuIrO}_{9}$ \\
\hline $\mathrm{Ba} 1-\mathrm{O} 1(\times 3)$ & $2.9433(5)$ & $2.9810(9)$ & $2.9484(9)$ & $2.9713(8)$ \\
$\mathrm{Ba} 1-O 1(\times 3)$ & $2.9438(5)$ & $2.9816(9)$ & $2.9490(9)$ & $2.9719(8)$ \\
$\mathrm{Ba} 1-\mathrm{O} 2(\times 6)$ & $2.920(13)$ & $2.973(14)$ & $2.987(12)$ & $2.963(13)$ \\
$\mathrm{Ba} 2-\mathrm{O} 1(\times 3)$ & $2.772(9)$ & $2.722(13)$ & $2.711(10)$ & $2.730(10)$ \\
$\mathrm{Ba} 2-O 2(\times 3)$ & $2.9452(6)$ & $2.9862(10)$ & $2.9508(7)$ & $2.9733(7)$ \\
$\mathrm{Ba} 2-O 2(\times 3)$ & $2.9458(6)$ & $2.9868(10)$ & $2.9514(7)$ & $2.9739(7)$ \\
$\mathrm{Ba} 2-O 2(\times 3)$ & $3.144(13)$ & $3.402(14)$ & $3.137(12)$ & $3.260(12)$ \\
$\mathrm{M}-\mathrm{O} 2(\times 6)$ & $2.093(14)$ & $2.240(15)$ & $2.150(13)$ & $2.197(14)$ \\
$\mathrm{Ru} / \mathrm{Ir}-\mathrm{O} 1(\times 3)$ & $2.194(12)$ & $2.232(18)$ & $2.237(14)$ & $2.237(14)$ \\
$\mathrm{Ru} / \mathrm{Ir}-\mathrm{O} 2(\times 3)$ & $2.046(14)$ & $2.010(16)$ & $2.018(13)$ & $2.010(14)$ \\
$(\mathrm{Ru} / \mathrm{Ir})-(\mathrm{Ru} / \mathrm{Ir})$ & $2.556(4)$ & $2.565(5)$ & $2.498(4)$ & $2.536(4)$ \\
\hline
\end{tabular}

\begin{tabular}{|c|c|c|c|c|c|}
\hline & $\mathrm{Ba}_{3} \mathrm{SmRuIrO}_{9}$ & $\mathrm{Ba}_{3} \mathrm{EuRuIrO}_{9}$ & $\mathrm{Ba}_{3} \mathrm{GdRuIrO}_{9}$ & $\mathrm{Ba}_{3} \mathrm{TbRuIrO}_{9}$ & $\mathrm{Ba}_{3} \mathrm{DyRuIrO}_{9}$ \\
\hline $\mathrm{Ba} 1-\mathrm{O} 1(\times 3)$ & $2.9650(9)$ & $2.9629(10)$ & $2.9586(8)$ & $2.9236(5)$ & $2.9508(9)$ \\
\hline $\mathrm{Ba} 1-\mathrm{O} 1(\times 3)$ & $2.9656(9)$ & $2.9634(10)$ & $2.9592(8)$ & $2.9242(5)$ & $2.9513(9)$ \\
\hline $\mathrm{Ba} 1-\mathrm{O} 2(\times 6)$ & $2.972(12)$ & $2.950(12)$ & $2.951(14)$ & $2.901(14)$ & $2.918(13)$ \\
\hline $\mathrm{Ba} 2-\mathrm{O} 1(\times 3)$ & $2.732(10)$ & $2.724(10)$ & $2.741(10)$ & $2.766(11)$ & $2.732(10)$ \\
\hline $\mathrm{Ba} 2-\mathrm{O} 2(\times 3)$ & $2.9653(6)$ & $2.9616(5)$ & $2.9588(6)$ & $2.9244(5)$ & $2.9489(4)$ \\
\hline $\mathrm{Ba} 2-\mathrm{O} 2(\times 3)$ & $2.9659(6)$ & $2.9622(5)$ & $2.9594(6)$ & $2.9249(5)$ & $2.9496(4)$ \\
\hline $\mathrm{Ba} 2-\mathrm{O} 2(\times 3)$ & $3.193(12)$ & $3.193(12)$ & $3.176(14)$ & $3.115(14)$ & $3.164(13)$ \\
\hline M-O2 $(\times 6)$ & $2.201(13)$ & $2.158(13)$ & $2.160(15)$ & $2.093(15)$ & $2.126(14)$ \\
\hline $\mathrm{Ru} / \mathrm{Ir}-\mathrm{O} 1(\times 3)$ & $2.251(15)$ & $2.265(14)$ & $2.238(14)$ & $2.180(14)$ & $2.253(14)$ \\
\hline $\mathrm{Ru} / \mathrm{Ir}-\mathrm{O} 2(\times 3)$ & $1.984(13)$ & $2.020(13)$ & $2.011(15)$ & $2.022(15)$ & $2.024(14)$ \\
\hline$(\mathrm{Ru} / \mathrm{Ir})-(\mathrm{Ru} / \mathrm{Ir})$ & $2.538(4)$ & $2.540(4)$ & $2.538(4)$ & $2.532(4)$ & $2.549(4)$ \\
\hline & $\mathrm{Ba}_{3} \mathrm{HoRuIrO}_{9}$ & $\mathrm{Ba}_{3} \mathrm{ErRuIrO}_{9}$ & $\mathrm{Ba}_{3} \mathrm{TmRuIrO}_{9}$ & $\mathrm{Ba}_{3} \mathrm{YbRuIrO}_{9}$ & $\mathrm{Ba}_{3} \mathrm{LuRuIrO}_{9}$ \\
\hline $\mathrm{Ba} 1-\mathrm{O} 1(\times 3)$ & $2.9459(7)$ & $2.9399(5)$ & $2.9378(4)$ & $2.9362(9)$ & $2.9305(5)$ \\
\hline $\mathrm{Ba} 1-\mathrm{O} 1(\times 3)$ & $2.9465(7)$ & $2.9405(5)$ & $2.9383(4)$ & $2.9368(9)$ & $2.9311(5)$ \\
\hline $\mathrm{Ba} 1-\mathrm{O} 2(\times 6)$ & $2.922(11)$ & $2.915(13)$ & $2.966(11)$ & $2.917(11)$ & $2.898(12)$ \\
\hline $\mathrm{Ba} 2-\mathrm{O} 1(\times 3)$ & $2.746(9)$ & $2.767(9)$ & $2.883(8)$ & $2.731(9)$ & $2.768(8)$ \\
\hline $\mathrm{Ba} 2-\mathrm{O} 2(\times 3)$ & $2.9455(4)$ & $2.9403(4)$ & $2.9396(6)$ & $2.9333(4)$ & $2.92981(32)$ \\
\hline $\mathrm{Ba} 2-\mathrm{O} 2(\times 3)$ & $2.9461(4)$ & $2.9409(4)$ & $2.9402(6)$ & $2.9339(4)$ & $2.93039(32)$ \\
\hline $\mathrm{Ba} 2-\mathrm{O} 2(\times 3)$ & $3.146(11)$ & $3.139(13)$ & $3.078(11)$ & $3.103(11)$ & $3.113(12)$ \\
\hline $\mathrm{M}-\mathrm{O} 2(\times 6)$ & $2.118(12)$ & $2.141(14)$ & $2.175(11)$ & $2.119(12)$ & $2.123(12)$ \\
\hline $\mathrm{Ru} / \mathrm{Ir}-\mathrm{O} 1(\times 3)$ & $2.232(12)$ & $2.194(12)$ & $2.041(9)$ & $2.255(13)$ & $2.202(11)$ \\
\hline $\mathrm{Ru} / \mathrm{Ir}-\mathrm{O} 2(\times 3)$ & $2.023(12)$ & $1.996(14)$ & $1.954(11)$ & $2.000(12)$ & $1.991(12)$ \\
\hline$(\mathrm{Ru} / \mathrm{Ir})-(\mathrm{Ru} / \mathrm{Ir})$ & $2.554(3)$ & $2.541(4)$ & $2.538(4)$ & $2.553(4)$ & $2.556(3)$ \\
\hline
\end{tabular}


Figure A - Field dependence of the magnetic susceptibility at $2 \mathrm{~K}$ (open circles) and 125 $\mathrm{K}$ (filled circles) for $\mathrm{Ba}_{3} \mathrm{NdRuIrO}_{9}$.

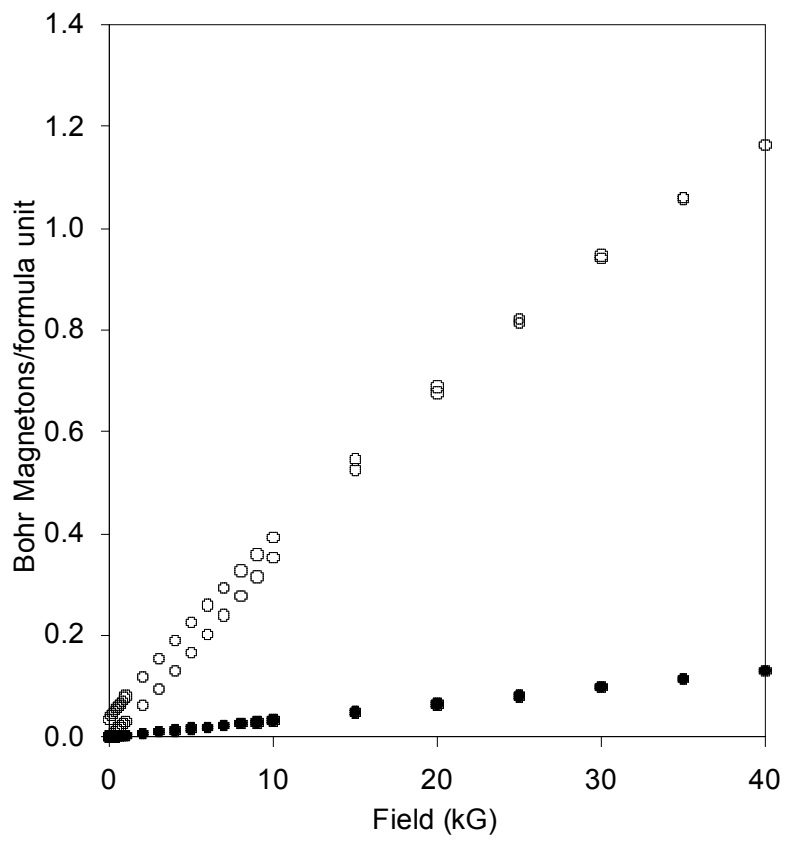

Table C - Magnetic moment for free $\operatorname{Ln}^{3+}\left(\mu_{\mathrm{Ln}^{3+}}{ }^{3+}\right.$, calculated magnetic moments using each magnetic ion $\left(\mathrm{Ru}^{5+} / \mathrm{Ir}^{4+}\right)\left(\mu_{\mathrm{cal}}\right)$, effective magnetic moments at $300 \mathrm{~K}\left(\mu_{\mathrm{eff}}\right)$, modified Curie Weiss fitting results in the temperature range 100-300 K.

\begin{tabular}{|c|c|c|c|c|c|c|c|}
\hline & $\mu_{\text {eff }} L n^{3+}$ & $\begin{array}{l}\mu_{\text {eff }}\left(\mu_{\mathrm{B}}\right) \\
(300 \mathrm{~K})\end{array}$ & $\mu_{\mathrm{eff}}\left(\mu_{\mathrm{B}}\right)(2 \mathrm{~K})$ & $\mu_{\mathrm{eff}}\left(\mu_{\mathrm{B}}\right)$ (fit) & $\begin{array}{l}\mu_{\mathrm{cal}} 300 \mathrm{~K}\left(\mu_{\mathrm{B}}\right) \\
\left(\mathrm{Ru}^{5+} / \mathrm{Ir}^{4+}\right)\end{array}$ & $\begin{array}{l}\mu_{\mathrm{cal}} 300 \mathrm{~K}\left(\mu_{\mathrm{B}}\right) \\
\left(\mathrm{Ru}^{4+} / \mathrm{Ir}^{5+}\right)\end{array}$ & $\begin{array}{l}\mu_{\mathrm{cal}} 2 \mathrm{~K} \\
\left(\mu_{\mathrm{B}}\right) \\
\left(\mathrm{Ru}^{4+} / \mathrm{Ir}^{5+}\right)\end{array}$ \\
\hline $\mathrm{La}$ & 0.0 & 2.46 & 0.47 & $2.27(3)$ & 2.29 & 3.08 & 2.83 \\
\hline $\mathrm{Nd}$ & 3.62 & 4.46 & 1.62 & $4.044(6)$ & 4.28 & 4.76 & 4.59 \\
\hline $\operatorname{Pr}$ & - & 2.42 & 0.47 & $1.66(2)$ & - & - & - \\
\hline $\mathrm{Sm}$ & 1.55 & 2.91 & 0.48 & $2.18(2)$ & 2.77 & 3.45 & 3.23 \\
\hline $\mathrm{Eu}$ & 3.40 & 3.98 & 0.43 & - & 4.10 & 4.59 & 4.42 \\
\hline Gd & 7.94 & 8.21 & 5.33 & $7.87(2)$ & 8.26 & 8.52 & 8.43 \\
\hline $\mathrm{Tb}$ & - & 8.35 & 2.50 & $8.22(1)$ & - & - & - \\
\hline Dy & 10.63 & 10.77 & 6.61 & $10.52(1)$ & 10.87 & 11.07 & 11.00 \\
\hline Ho & 10.58 & 10.90 & 5.79 & $10.70(2)$ & 10.83 & 11.02 & 10.95 \\
\hline $\mathrm{Er}$ & 9.59 & 9.88 & 5.91 & $9.87(1)$ & 9.86 & 10.07 & 10.00 \\
\hline $\mathrm{Tm}$ & 7.55 & 7.95 & 1.51 & $8.23(2)$ & 7.89 & 8.16 & 8.06 \\
\hline $\mathrm{Yb}$ & 4.54 & 4.72 & 1.71 & $4.88(4)$ & 5.09 & 5.49 & 5.35 \\
\hline $\mathrm{Lu}$ & 0.0 & 2.25 & 0.34 & $2.87(2)$ & 2.29 & 3.08 & 2.83 \\
\hline $\mathrm{Y}$ & 0.0 & 2.73 & 0.40 & $2.23(2)$ & 2.29 & 3.08 & 2.83 \\
\hline
\end{tabular}


Table D - Fitting results using a dimer model, where $\mathrm{g}$ is the gyromagnetic ratio, $\mathrm{J}$ is the exchange interaction term, and $x$ is the dimer fraction term in $(1-2 x)\left(\operatorname{Ir}^{4+} / \mathrm{Ru}^{5+}\right)+$ $(x)\left(\mathrm{Ir}^{4+} / \mathrm{Ir}^{4+}\right)+(x)\left(\mathrm{Ru}^{5+} / \mathrm{Ru}^{5+}\right)$.

\begin{tabular}{llll}
\hline & $\mathrm{g}$ & $\mathrm{J}(\mathrm{K})$ & $\mathrm{x}$ \\
\hline $\mathrm{Ba}_{3} \mathrm{LuRuIrO}_{9}$ & $2.018(6)$ & $-42.3(3)$ & $0.3853(6)$ \\
$\mathrm{Ba}_{3}$ YRuIrO $_{9}$ & $1.488(5)$ & $-43.3(4)$ & $0.4058(6)$ \\
$\mathrm{Ba}_{3}$ LaRuIrO $_{9}$ & $1.159(4)$ & $-20.0(3)$ & $0.382(2)$ \\
\hline
\end{tabular}

